\author{
Sebastian Buhl ${ }^{\mathrm{a}, \mathrm{b}}$, Christian Greiner ${ }^{\mathrm{a}, \mathrm{c}}$, Aránzazu del Campo ${ }^{\mathrm{a}, \mathrm{d}}$, Eduard Arzt ${ }^{\mathrm{d}}$ \\ ${ }^{a}$ Max Planck Institute for Metals Research, Stuttgart, Germany \\ ${ }^{\mathrm{b}}$ now at: ETH Zurich, IWF, Zurich, Switzerland and EMPA, LJIT, Duebendorf, Switzerland \\ ${ }^{c}$ now at: University of Pennsylvania, Department of Mechanical Engineering and Applied Mechanics, Philadelphia, USA \\ ${ }^{\mathrm{d}}$ INM - Leibniz Institute for New Materials, Saarbruecken, Germany
}

\title{
Humidity influence on the adhesion of biomimetic fibrillar surfaces
}

\author{
Dedicated to Professor Dr. Franz Jeglitsch on the occasion of his $75^{\text {th }}$ birthday
}

\begin{abstract}
The recent interest in fibrillar biological attachment systems, as found in the gecko, has led to the development of micropatterned elastomer adhesion surfaces. All reported studies have been performed at ambient humidity neglecting its possible influence on adhesion. The present paper investigates, for the first time, the effect of systematic changes in ambient humidity from 2 to $90 \%$. Adhesion measurements were performed on PDMS (Sylgard 184) surfaces possessing micropillars with flat-ended and hemispherical contact shape. The pillar radius was varied between 2.5 and $25 \mu \mathrm{m}$; the pillar aspect ratio was kept at 1 . While the adhesion of a flat sample was not affected by humidity, we found that pillar size and shape influenced the sensitivity to humidity changes: Thinner pillars, with higher pull-off forces in the dry state, exhibited decreasing adhesion values, by up to $35 \%$, with increasing humidity. The effect was stronger for the hemispherical tip shape, where the positive effect of finer pillars was even reversed. Possible explanations for these effects, which may lower the reliability of biomimetic adhesion devices in the presence of humidity, are given.
\end{abstract}

Keywords: Adhesion; Patterned surfaces; Gecko adhesive; Humidity; Shape

\section{Introduction}

Bio-inspired fibrillar adhesives have sparked much interest lately, as they promise to mimic the fascinating adhesive properties found in animals such as the gecko. If successful, they would stick to any surface, be completely reversible as well as self-cleaning. The parameter space in designing such adhesives is vast and current progress in this field was recently reviewed by Chan et al. [1] and del Campo and Arzt [2]. One of the attempts to guide the design of fibrillar adhesives and to visualize critical design criteria was brought forward by Spolenak et al. [3] in the concept of adhesion design maps, lately extended by Greiner et al. [4]. Among the design parameters so far studied experimentally, are the influence of contact size $[5,6]$ contact shape $[7,8]$, backing layer thickness $[9,10]$, Young's Modulus [10], fiber aspect ratio [5] and hierarchy [11, 12]. A factor which has so far been widely neglected is the influence of ambient humidity on the performance of such fibrillar systems. This might be an important parameter as, when investigating gecko spatula, Huber et al. [13] found an increase in adhesion with humidity. A similar effect was observed by Sun et al. [14], providing experimental data to support the role of capillary contributions to gecko adhesion.

In the present paper, the influence of ambient humidity on the adhesion performance of bio-inspired micropatterned surfaces was studied. We performed our experiments for two contact shapes, which are known to exert a significant influence on adhesion [7]. The shapes studied are flat punch-like and hemispherical. The aim of the study is to advance the understanding of how these adhesives work, and to lay a foundation for ensuring reliable adhesion in future commercial use where a wide range of humidities will be encountered.

\section{Experimental}

Arrays of PDMS micro pillars were obtained by soft-molding Sylgard 184 on arrays of holes made by lithographic patterning of thick SU-8 films, as reported previously [5, $15,16]$. The radii of the fabricated pillars varied from $r=2.5$ to $25 \mu \mathrm{m}$, with identical height, yielding an aspect ratio $\lambda$ of 1 . The interpillar distance was the same as the pillar diameter and the packing geometry was hexagonal, resulting in an areal packing density of $22.7 \%$. Silicon wafers (100 orientation) were provided by Crystec (Berlin, Germany). SU-8 photo resists and the developer mr-Dev 600 were provided by Micro Resist Technology (Berlin, Germany). Hexadecafluoro-1,1,2,2,-tetrahydrooctyltrichlorosilane was purchased from ABCR (Karlsruhe, Germany). Masks for lithography were provided by ML\&C (Jena, Germany) in quartz with $0.8 \times 0.8 \mathrm{~cm}^{2}$ chrome patterned fields. A mask aligner Karl Suss MJB3 (Garching, Germany) was used for the irradiation step. Sylgard 184 was purchased from Dow Corning (MI, USA). The profiles of the patterned surfaces were characterized by interferometry using a ZYGOLOT 5000 equipment and by scanning electron microscopy using a LEO 1530VP.

Soft-molding: A 10:1 ratio of Sylgard 184 prepolymer and crosslinker was mixed, degassed and poured on a silanized SU-8 patterned wafer (see Ref. [5] for more details). For all samples, curing for $14 \mathrm{~h}$ at $65^{\circ} \mathrm{C}$ in light vacuum ( $\sim 600 \mathrm{mbar}$ ) afforded accurate replicas. The total thickness of the elastomer samples was controlled with a Teflon ring of defined height surrounding the wafer. All samples had a sample thickness of $5 \mathrm{~mm}$. This procedure gave pillars with a flat punch-like contact shape. To fabricate pillars with spherical terminals, we used a method based on inking with uncured PDMS precursor (see Refs. [7, 16] for more 
S. Buhl et al.: Humidity influence on the adhesion of biomimetic fibrillar surfaces

details): A 1:10 ratio of Sylgard 184 prepolymer and crosslinker was mixed, degassed and spin-coated onto a silicon wafer to obtain film thicknesses below $6 \mu \mathrm{m}$. Previously soft-molded arrays of PDMS pillars were inked in the spin-coated film and cured upside-down in an oven for $14 \mathrm{~h}$ at $65^{\circ} \mathrm{C}$. A nearly hemispherical cap formed at the top of the pillars as a consequence of gravity and surface tension.

Adhesion measurements: The adhesion performance of the hexagonal patterned surfaces was tested by recording load-displacement curves obtained with a home-built indentation equipment $[5,7,17]$. This apparatus consisted of a glass spring mounted on a piezoelectric crystal (P-611 NanoCube from Physik Instrumente, Karlsruhe, Germany) and coupled to a hexapod nanopositioning stage (F-206 from Physik Instrumente, Karlsruhe, Germany). A single crystal sapphire sphere with a diameter of $5 \mathrm{~mm}$ and a high-precision polished surface provided by Goodfellow (Huntingdon, U.K.) was glued to the free end of the glass spring. Using a spherical tip solved the problem of possible misalignment between probe and surface occurring in flatflat contacts. The patterned sample was placed on the positioning stage and the sphere was brought into contact. After compressive preloading, the sphere was retracted at a constant rate of $1 \mathrm{\mu m} \mathrm{s}^{-1}$ until pull-off occurred. The maximum vertical displacement of the piezo was $100 \mu \mathrm{m}$ and the positioning accuracy was $1 \mathrm{~nm}$. The stage had a maximum travel range of $12 \mathrm{~mm}$ with an accuracy of $100 \mathrm{~nm}$. Spring deflection during contact was monitored via a laser interferometer (SP 120 from SIOS, Ilmenau, Germany). Calibration of the spring allowed conversion of the deflection into force. For all tests, a four-leaf force sensing spring was installed in the equipment. More details on this spring design can be found in Ref. [18]; the spring had a stiffness of $309 \mathrm{~N} \mathrm{~m}^{-1}$. Data collection was performed with a Labview software package. The sapphire sphere was cleaned with high-purity ethanol before each test.

Since the mechanical properties of polymers may change over time, all adhesion experiments were performed immediately after fabrication without any further aging of the sample. The laboratory temperature and the humidity were registered for each measurement with a P 330 tool from Tematec (Hennef, Germany). For measuring the adhesion properties under different ambient humidities, the entire setup was encapsulated within a PMMA box. The box was either flushed with dry nitrogen or with nitrogen after being pressed through water in a washing flask at different flow rates. For humidities exceeding $60 \%$, the water was heated up to about $90^{\circ} \mathrm{C}$. The accuracy of this method was within $2 \%$ points in relative humidity. Before starting an adhesion experiment, the humidity was equilibrated for $1 \mathrm{~h}$, which in preliminary tests had proven to be the optimum delay time. A minimum of 5 measurements was performed for each data point and after each experiment, a new measurement location was chosen to circumvent any effect of repetitive measurements at the same spot (see Ref. [10]). For comparison, adhesion tests under the same conditions were also conducted with flat, unstructured specimens.

\section{Results}

Representative scanning electron microscope (SEM) pictures showing the quality of our samples are depicted in
Fig. 1. The patterned fields $\left(0.8 \times 0.8 \mathrm{~mm}^{2}\right)$ were defect free, as required for reproducible measurements. It is known in the literature that in the case of testing fibrillar adhesives with a spherical indenter, the adhesive behavior is a function of the maximum compressive preload $P_{\mathrm{p}}$ [5]. The most common descriptor for such adhesives is the pull-off force $P_{\mathrm{c}}$, which in our case is the force necessary to separate the sapphire sphere from the elastomer sample. As the pulloff force will be a function of indenter geometry, another meaningful way to characterize adhesion is the pull-off strength $\sigma_{\mathrm{c}}$, which is evaluated by dividing the pull-off force by the (calculated) apparent contact area of the indenting sphere with the patterned surface, at maximum preload $[5,19]$. Typical plots for the pull-off force $P_{\mathrm{c}}$ and the pull-off strength $\sigma_{\mathrm{c}}$ as functions of preload $P_{\mathrm{p}}$ are shown in Fig. 2 using the example of flat punch-shaped pillars with a radius of $5 \mu \mathrm{m}$. The full curves for $P_{\mathrm{c}}$ and $\sigma_{\mathrm{c}}$ as functions of the preload for all other tested samples can be found in the Appendix. To ease comparison and to highlight the effect of humidity, we plot the pull-off force (Fig. 3a) and the pull-off strength (Fig. 3b) for flat punch-shaped micropillars with four different pillar radii between 2.5 and $25 \mu \mathrm{m}$ as a function of relative humidity $R H$ at a constant preload of $P_{\mathrm{p}}=2.0 \mathrm{mN}$. This approach of using a fixed preload has previously proved its merit in the study of other de-
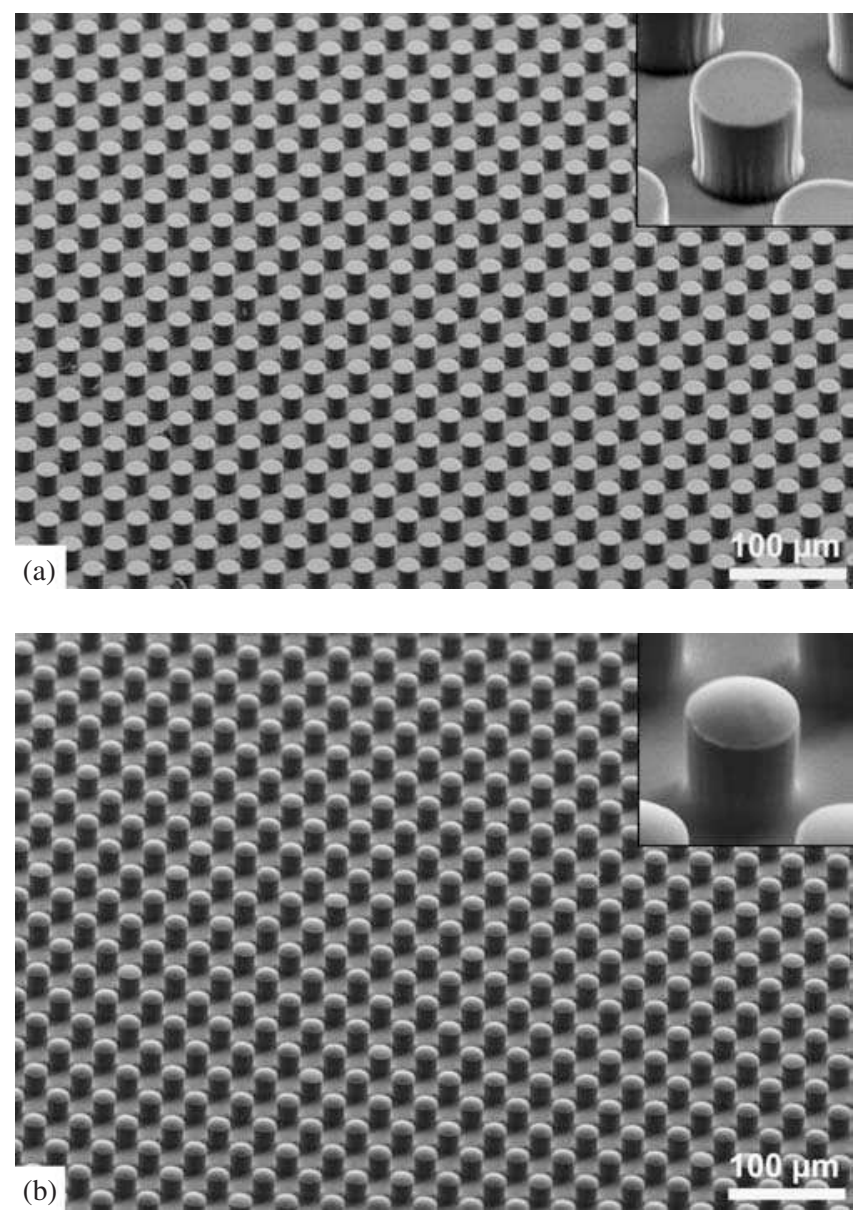

Fig. 1. SEM micrographs of PDMS test surfaces structured with $10 \mu \mathrm{m}$ radius pillars having an aspect ratio of 1 (hexagonal packing, packing density $22.7 \%$ ). (a) Flat punch-like and (b) hemispherical contact shape. The specimens were coated with $10 \mathrm{~nm}$ of $\mathrm{Au} / \mathrm{Pd}$ for SEM observation.

Int. J. Mat. Res. (formerly Z. Metallkd.) 100 (2009) 8 
S. Buhl et al.: Humidity influence on the adhesion of biomimetic fibrillar surfaces

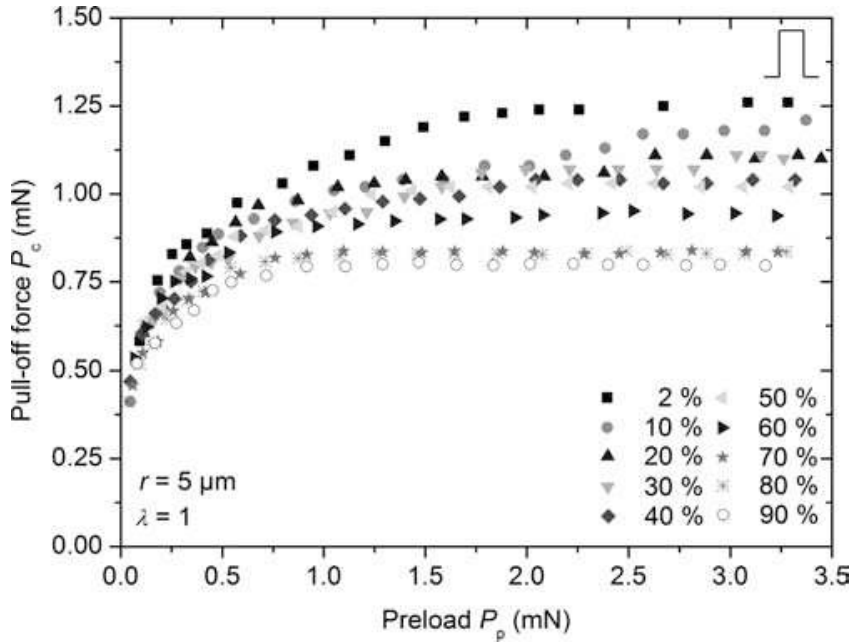

(a)

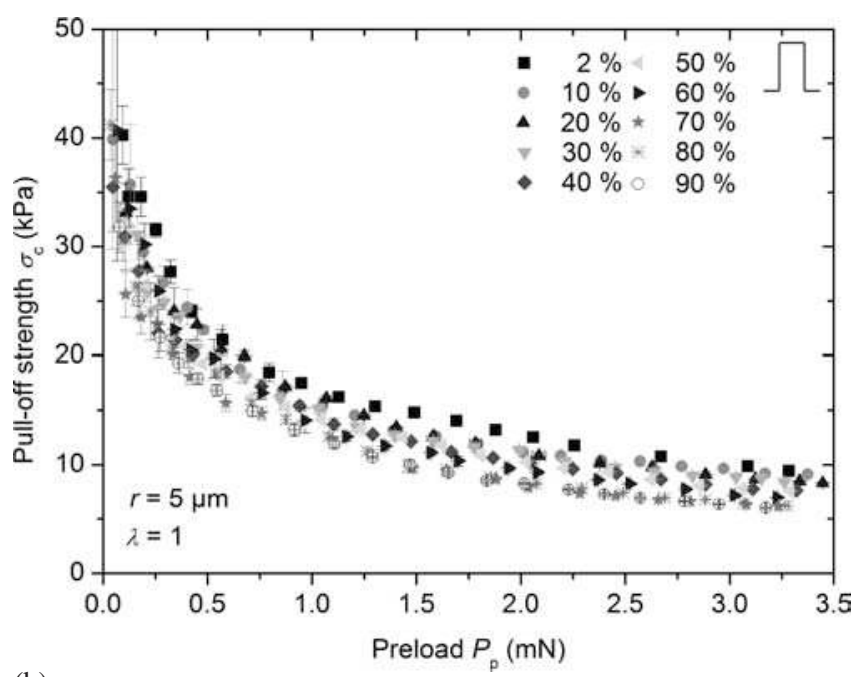

(b)

Fig. 2. Effect of humidity: (a) Pull-off force vs. preload, (b) pull-off strength $\sigma_{\mathrm{c}}$ (force per apparent contact area) vs. preload, for flat punch-shaped pillars with a radius of $r=5 \mu \mathrm{m}$ and an aspect ratio of 1 .

sign parameters $[5,7,10]$. Figure 3 indicates that for a pillar radius of 25 and $10 \mu \mathrm{m}$ there is no influence of humidity on adhesion, whereas it decreases with $R H$ for $r=5$ and $2.5 \mu \mathrm{m}$. For the $5 \mu \mathrm{m}$ radius pillars the decrease was around $35 \%$ and for $r=2.5 \mu \mathrm{m}, 19 \%$. Interestingly, the behavior, and also the percentage of the adhesion performance decrease, was very similar for both quantities in Fig. 3. It is emphasized that the decreases occur only for the pillar structures with the higher adhesion values, which were found for the finer pillars as expected [5].

To investigate whether this behavior is only found for flat punch-like structures, we performed similar tests with samples showing hemispherical tip shape. The results of these experiments are presented in Fig. 4. The diagrams show a similar behavior as for the flat punches: For the largest radius $(r=25 \mu \mathrm{m})$, adhesion is independent of humidity, whereas it decreases with $R H$ for the two smallest ones $(r=5$ and $2.5 \mu \mathrm{m})$. The decrease is less pronounced than with the flat punch-like structures: $26 \%$ for $r=10 \mu \mathrm{m}$ and $18 \%$ for $r=2.5 \mu \mathrm{m}$. In contrast to the results in Fig. 3, for a pillar radius of $10 \mu \mathrm{m}$, a $25 \%$ decrease with humidity

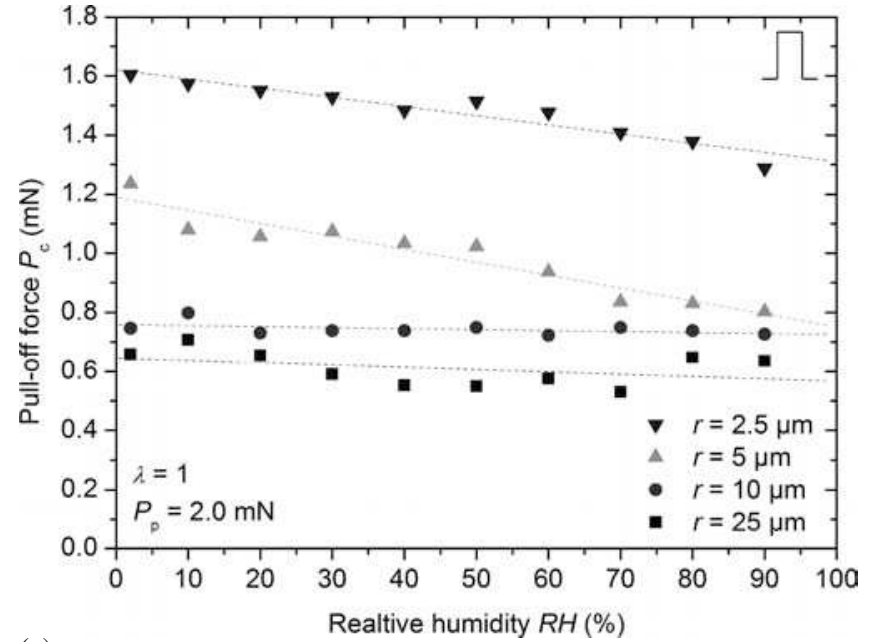

(a)

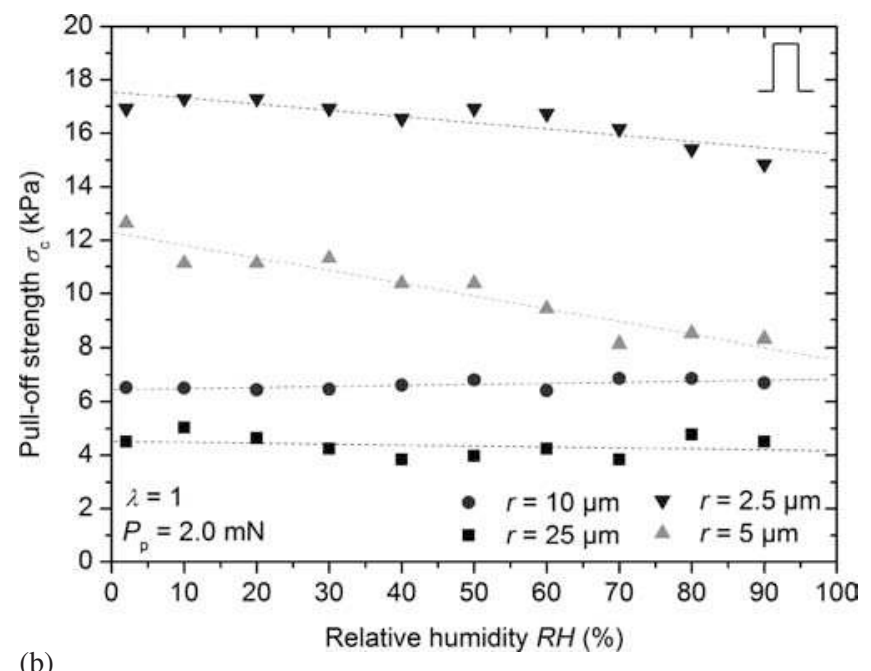

Fig. 3. Humidity effect for flat punch-like pillars: Pull-off force and strength at ten different ambient humidities between 2 and $90 \%$. The humidity was equilibrated for $1 \mathrm{~h}$. (a) Plotted as pull-off force vs. preload, (b) pull-off strength vs. relative humidity (at a preload of $2 \mathrm{mN}$ ). The broken lines indicate linear fits to the data.

was found. As with the flat punches, pull-off force and strength showed very similar behavior.

For comparison, the adhesion behavior of flat, unstructured PDMS is shown in Fig. 5; no significant influence of humidity is detected for these control samples.

\section{Discussion}

In this paper, we report on the first systematic study of humidity effects on the adhesion of bio-inspired fibrillar surfaces. We investigated two different tip shapes: flat punchlike and hemispherical. From the results presented above, it is clear that both fiber radius and shape seem to determine to what extent humidity influences the adhesion behavior; fine pillars and spherical shapes show an increased sensitivity to humidity. In the following, we discuss the results in detail.

The reference behavior is that of flat, unstructured PDMS, as presented in Fig. 5. These data demonstrate that there was no significant influence of ambient humidity on the pull-off force, or the pull-off strength, over a range in 
S. Buhl et al.: Humidity influence on the adhesion of biomimetic fibrillar surfaces

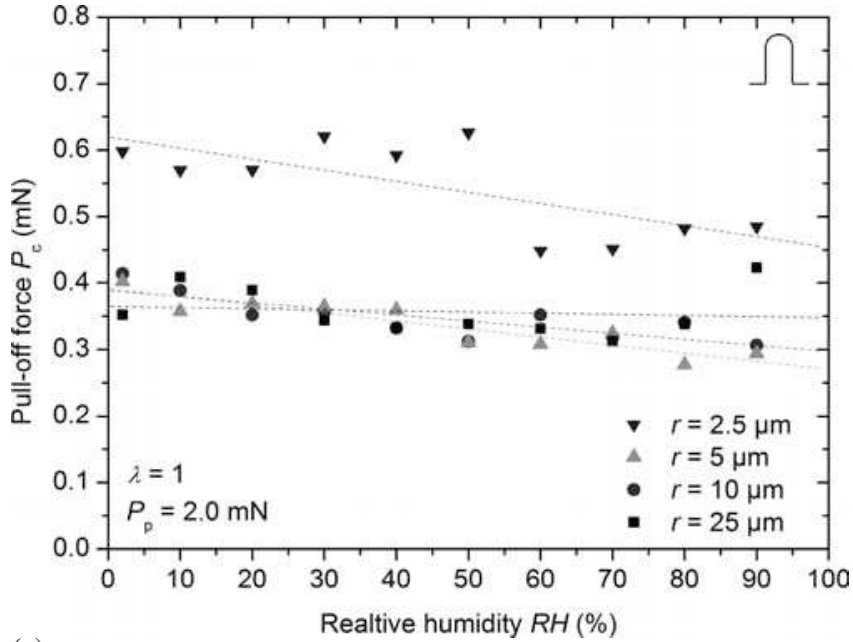

(a)

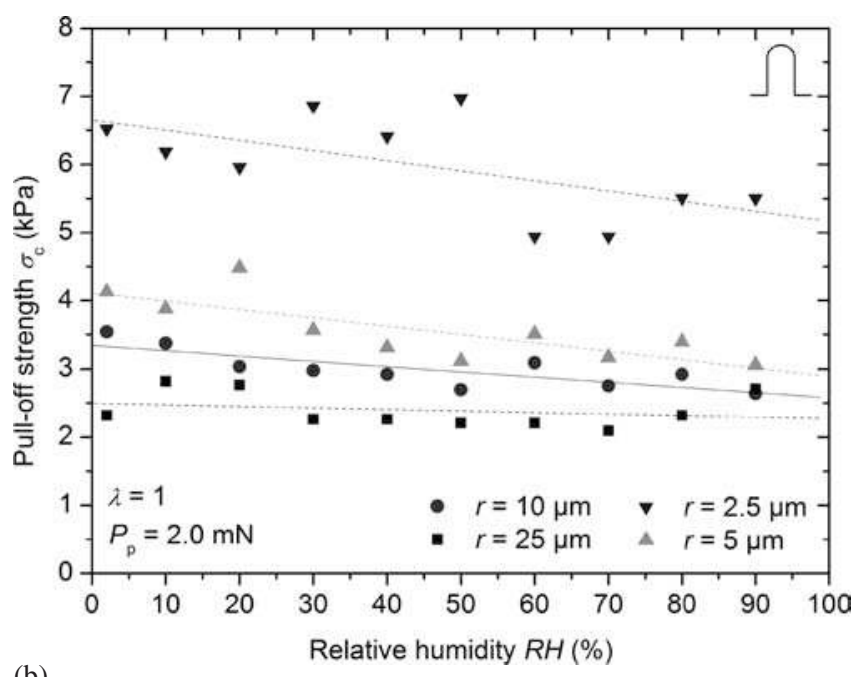

(b)

Fig. 4. Same as Fig. 3 for hemispherical tip shape.

RH from $2 \%$ to $90 \%$. This behavior can be explained by the hydrophobic nature of PDMS (water contact angle $\sim 108^{\circ}$ ). It will therefore not attract condensed water vapor from the ambient atmosphere, making the contribution of capillary forces unlikely. It is noted that the literature seems inconsistent when it comes to the effect of humidity on adhesion, even for similar contact pairs as in our experiments [2027]. Some authors $[21-23,26,27]$ report an increase in adhesion, some a decrease [25] and others find constant values [23, 26, 27] with increasing humidity. For PDMS, humidity effects have been invoked as an adhesionenhancing mechanism only in plasma treated form [20]; for untreated PDMS the same authors [20] found a behavior very similar to the one reported here. For the two larger radii $(r=25$ and $10 \mu \mathrm{m})$ with the flat punch-like fibers and for $r=25 \mu \mathrm{m}$ in the case of the hemispheres, we found no effect of humidity, which again can be attributed to the hydrophobic nature of PDMS. The wettability properties of the sapphire sphere did not influence the adhesion results, so that all subsequent discussed changes are attributed to the structuring of the PDMS surface.

Interestingly, for single gecko spatulae in contact with a hydrophilic substrate possessing a similar contact angle to water as our samples, Huber et al. [13] found increasing

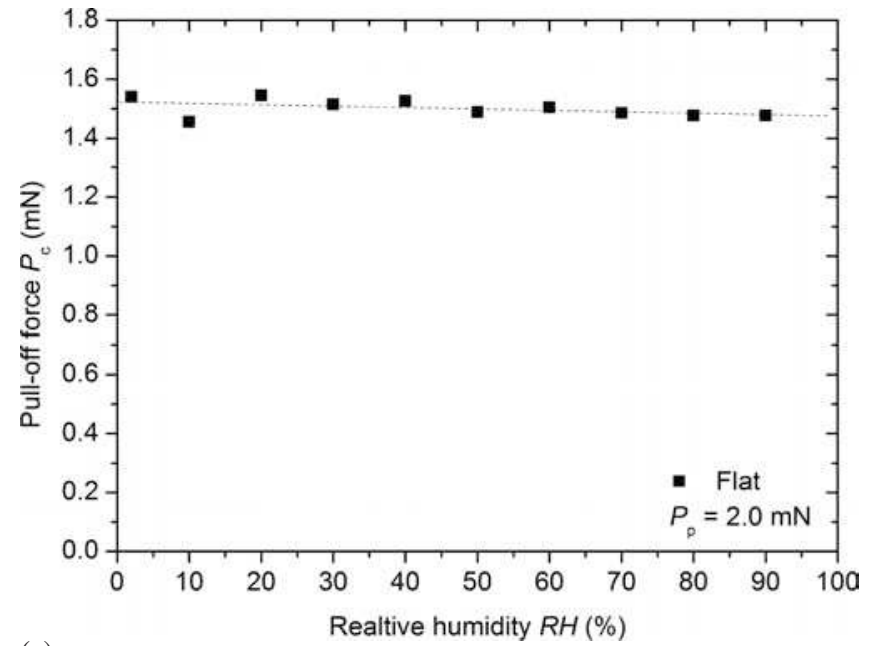

(a)

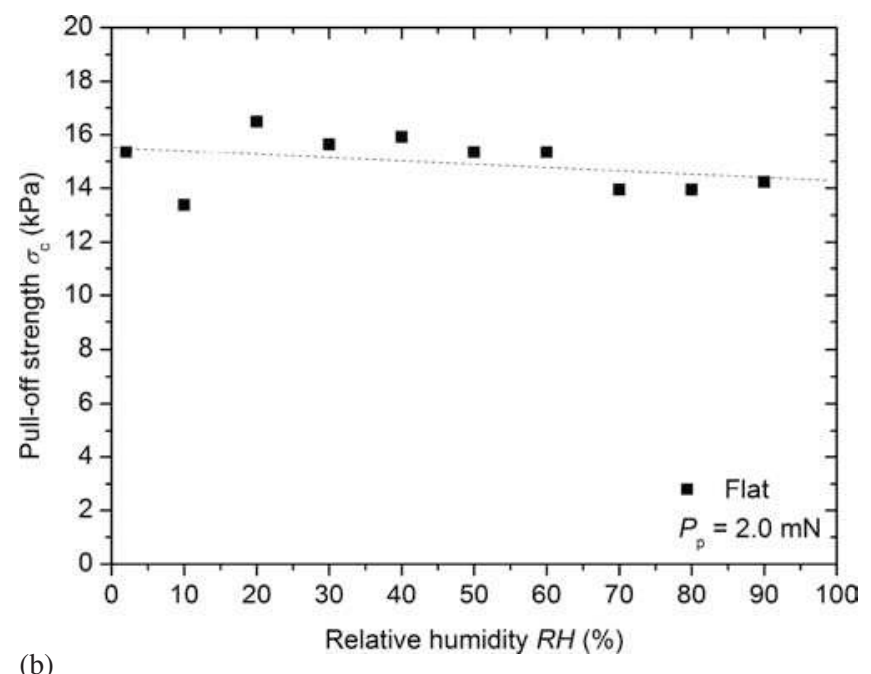

Fig. 5. Same as Fig. 3 for flat PDMS.

pull-off forces with increasing humidity. This was attributed to a monolayer of water adsorbed on the slightly rough substrate causing additional contact area [13]. It is important to note that the fibers in our samples are in the micron range ( 2.5 to $25 \mu \mathrm{m}$ diameter), whereas the gecko spatulae investigated by Huber et al. were much finer (about $200 \mathrm{~nm}$ ). It might thus not be surprising that such an effect was not found with the flat substrates in our experiments.

This argumentation still leaves the question of why for smaller radii, adhesion decreases with humidity. Pohlmann and zum Gahr [25] investigated the adhesion between silicon and a pre-oxidized $\mathrm{SiC}$ ceramic by atomic force microscopy (AFM). They also reported a decrease in adhesion force with increasing humidity. To explain this trend, they argued that, with increasing humidity, more of the surface was covered with water molecules, which lowered the surface energy and thus the effective work of adhesion. The same trend was reported by Bailey and Kay [28] for the interfacial energy between mica and water with increasing $R H$. If a similar process occurred with our samples, both the size and the shape effects might be tentatively explained as follows. As PDMS is hydrophobic compared to the hydrophilic SiC, condensation of water molecules is unlikely, but with a sufficient number of small surface features and crack-like struc- 
tures capillary condensation [29, 30] might take place. At a temperature of $20^{\circ} \mathrm{C}$, we calculate Kelvin radii $r_{\mathrm{K}}$ for water between $r_{\mathrm{K}}=-0.5 \mathrm{~nm}$ for $R H=10 \%$ and $r_{\mathrm{K}}=-10 \mathrm{~nm}$ for $R H=90 \%$, so that for small enough features capillary condensation may occur. Such a small amount of water molecules on the samples will lower the surface energy and thereby reduce the adhesion force. The amount of water condensing will not be enough to form liquid bridges, which through capillary forces would increase the pull-off force. That capillary condensation may take place with hydrophobic surfaces was also found by Cheng et al. [31] and by Wier and McCarthy [32], supporting our argumentation. Furthermore, water molecules between the PDMS sample and the sapphire sphere will lower the Hamaker constant [33] and thereby lead to a decease in van der Waals forces which are made responsible for adhesion with our samples.

Such an explanation is also consistent with the observed shape effect, according to which an influence of humidity for pillars of $10 \mu \mathrm{m}$ radius is only found with hemispherical tip shape. We attribute this to the fact that capillary condensation will be more likely in the small crack-like features near the contacts between the sphere and the hemispherical PDMS pillars. Thus it stands to reason that this shape will exhibit more humidity influence than the punch shape.

Also the size effect can be discussed in this context. In previous studies [5, 7] we demonstrated in detail the effect of contact splitting and concluded that - for most contact shapes investigated - smaller pillars yield higher adhesion. This might not to be the case when a varying ambient humidity is considered. The data presented in this study show that the smaller features are more susceptible to a reduction in the adhesion performance with increasing humidity. One explanation for this finding goes as follows: With smaller pillars, more small and crack-like surface features will be found between the sample surface and the sapphire sphere used for testing. This statistical effect will lead to more water molecules attracted from the ambient atmosphere through capillary condensation, lowering the Hamaker constant and the work of adhesion. Ultimately, this yields a stronger humidity influence on the adhesion of smaller pillars.

This negative size effect due to humidity may have unexpected practical consequences: Finer pillar structures will be rendered less adhesive than coarser structures when humidity increases. In our data this unexpected cross-over is clearly visible in Fig. 4a, where the adhesive force of $10 \mu \mathrm{m}$ pillars eventually falls below that of $25 \mu \mathrm{m}$ pillars when the humidity exceeds $\approx 30 \%$. Humidity effects may thus limit the reliable performance of biomimetic fibrillar adhesives under practical conditions which will include unexpected changes in humidity.

\section{Conclusion}

Sylgard 184 surfaces were micropatterned with flat-ended and hemispherical pillars of $25,10,5$ and $2.5 \mu \mathrm{m}$ radius and an aspect ratio of 1 . The adhesion properties of these surfaces and of flat, unstructured PDMS were measured with a spherical probe indenter and the influence of ambient humidities between 2 and $90 \%$ was investigated. We found effects of pillar size and shape on the susceptibility to humidity effects as follows:

- Flat and unstructured PDMS: No effect of ambient humidity was found between 2 and $90 \%$.
- Pillar radius of $25 \mu \mathrm{m}$ : Humidity did not influence the adhesion performance for both tip shapes.

- Pillar radius of $10 \mu \mathrm{m}$ : The adhesion behavior depended on the tip shape. For flat punch-like pillars there was no effect, whereas for the hemispherical tip shape pull-off force and strength decreased by about $25 \%$ at the maximum relative humidity of $90 \%$.

- Pillar radius of 5 and $2.5 \mu \mathrm{m}$ : For both tip shapes the adhesion performance dropped significantly with increasing humidity. The strongest decrease was found with the $5 \mu \mathrm{m}$ radius structures. In the case of the hemispherical tip shape the decrease was about $25 \%$. For the flat punches adhesion dropped by $35 \%$.

- The size-dependent influence of humidity on adhesion was attributed to effects of capillary condensation, which will be favored in smaller structures, lowering the surface energy and hence decreasing adhesion. This effect may counteract the beneficial effect of contact splitting, which favors small structures in the dry state. This could be important in commercial applications where reliable adhesion for all humidities has to be ensured.

Lithography was performed in the cleanroom of the Max Planck Institute for Solid State Research; we are grateful to Prof. K. von Klitzing and M. Riek. Part of this work was funded by the Volkswagen foundation.

\section{References}

[1] E.P. Chan, C. Greiner, E. Arzt, A.J. Crosby: MRS Bulletin 32 (2007) 496.

[2] A. Del Campo, E. Arzt: Macromol. Biosci. 7 (2007) 118.

[3] R. Spolenak, S. Gorb, E. Arzt: Acta Biomater. 1 (2005) 5.

[4] C. Greiner, R. Spolenak, E. Arzt: Acta Biomater. 5 (2009) 597.

[5] C. Greiner, A. Del Campo, E. Arzt: Langmuir 23 (2007) 3495.

[6] E. Arzt, S. Gorb, R. Spolenak: Proceedings of the National Academy of Sciences of the United States of America 100 (2003) 10603.

[7] A. Del Campo, C. Greiner, E. Arzt: Langmuir 23 (2007) 10235.

[8] S. Kim, M. Sitti: Appl. Phys. Lett. 89 (2006) 261911.

[9] S. Kim, M. Sitti, C.Y. Hui, R. Long, A. Jagota: Appl. Phys. Lett. 91 (2007).

[10] C. Greiner, S. Buhl, A. Del Campo, E. Arzt: The Journal of Adhesion, submitted for publication (2008).

[11] C. Greiner, E. Arzt, A. Del Campo: Adv. Mater. 21 (2009) 479

[12] M.T. Northen, K.L. Turner: Nanotechnology 16 (2005) 1159.

[13] G. Huber, H. Mantz, R. Spolenak, K. Mecke, K. Jacobs, S.N. Gorb, E. Arzt: Proceedings of the National Academy of Sciences of the United States of America 102 (2005) 16293.

[14] W.X. Sun, P. Neuzil, T.S. Kustandi, S. Oh, V.D. Samper: Biophys. J. 89 (2005) L14.

[15] A. Del Campo, C. Greiner: J. of Micromech. and Microeng. 17 (2007) R81.

[16] A. Del Campo, C. Greiner, I. Álvarez, E. Arzt: Adv. Mater. 19 (2007) 1973.

[17] A. Peressadko, N. Hosoda, B.N.J. Persson: Phys. Rev. Lett. 95 (2005) 124301.

[18] M. Varenberg, A. Peressadko, S. Gorb, E. Arzt, S. Mrotzek: Rev. of Sc. Instrum. 77 (2006).

[19] M.T. Northen, C. Greiner, E. Arzt, K.L. Turner: Adv. Mater. 20 (2008) 3905.

[20] B. Bhushan, M. Cichomski: J. of Vac. Sci., Technology A 25 (2007) 1285 .

[21] B. Bhushan, C. Dandavate: J. of Appl. Phys. 87 (2000) 1201.

[22] A. Ata, Y.I. Rabinovich, R.K. Singh: J. of Adhes. Sci. Technol. 16 (2002) 337.

[23] A. Fukunishi, Y. Mori: Adv. Powder Technol. 17 (2006) 567.

[24] D.B. Asay, S.H. Kim: J. of Chem. Phys. 124 (2006) 174712.

[25] K. Pohlmann, K.H.Z. Gahr: Materialwiss. Werkstofftech. 31 (2000) 280

[26] V. Turq, N. Ohmae, J.M. Martin, J. Fontaine, H. Kinoshita, J. Loubet: Tribol. Lett. 19 (2005) 23. 
S. Buhl et al.: Humidity influence on the adhesion of biomimetic fibrillar surfaces

[27] B. Hoffmann, B. Kubier: Chemie Ingenieur Technik 75 (2003) 742.

[28] A.I. Bailey, S.M. Kay: Proc. R. Soc. A 301 (1967) 47.

[29] K.-T. Wan, B.R. Lawn: Acta Metall. Mater 38 (1990) 2073.

[30] M. Ciccotti, M. George, V. Ranieri, L. Wondraczek, C. Marlière: J. Non-Cryst. Solids 354 (2008) 564.

[31] Y.-T. Cheng, D.E. Rodak, A. Angelopoulos, T. Gacek: Appl. Phys. Lett. 87 (2005) 194112.

[32] K.A. Wier, T. J. McCarthy: Langmuir 22 (2006) 2433.

[33] J.N. Israelachvili (1992) Intermolecular and Surface Forces (Academic Press, New York), pp. 188-192.

(Received January 22, 2009; accepted May 18, 2009)

\section{Bibliography}

DOI 10.3139/146.110146

Int. J. Mat. Res. (formerly Z. Metallkd.)

100 (2009) 8; page 1119-1126

(C) Carl Hanser Verlag GmbH \& Co. KG

ISSN 1862-5282

\section{Correspondence address}

Dr. Christian Greiner

University of Pennsylvania

Department of Mechanical Engineering and Applied Mechanics

112 Towne Building

220 South $33^{\text {rd }}$ St., Philadelphia, PA 19104-6315 USA

E-mail: cgreiner@seas.upenn.edu

\section{Appendix}

To allow for a full comparison between the different surface structures, we here present the full pull-off force and pull-off strength curves for all samples (except for the flat-punch shaped sample with $r=5 \mu \mathrm{m}$ which was presented in Fig. 2).

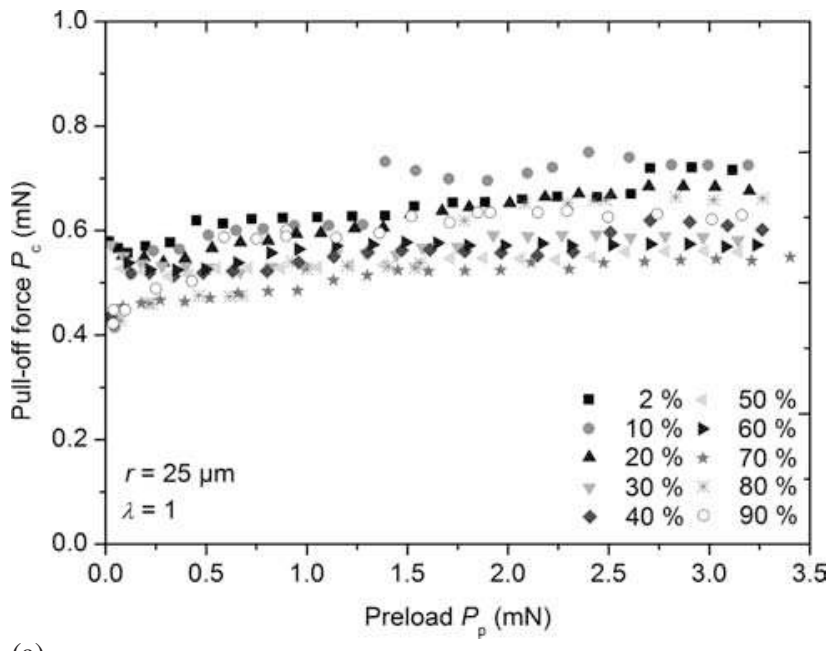

(a)

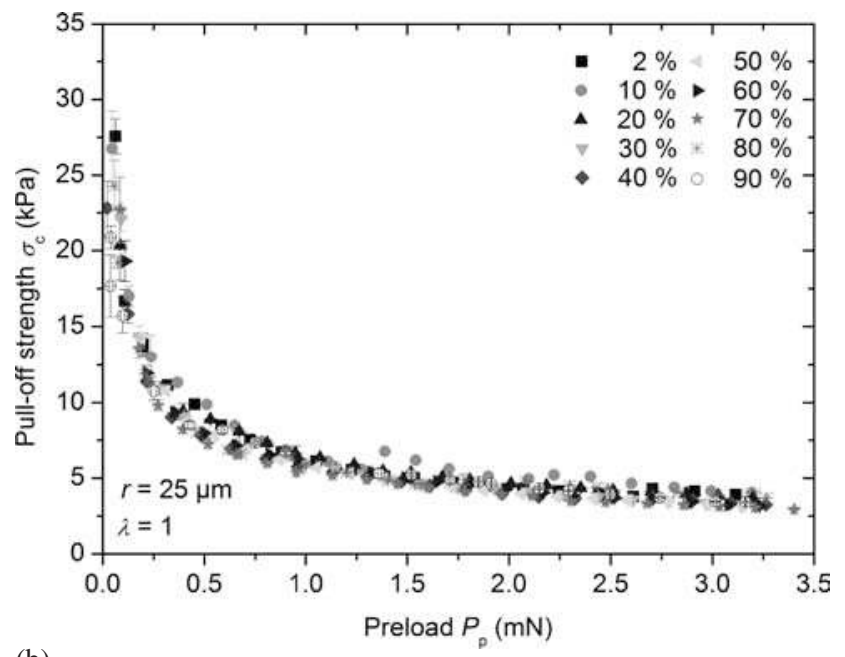

(b)

Fig. A1. Effect of ambient humidity on adhesion: (a) Pull-off force vs. preload, (b) pull-off strength $\sigma_{\mathrm{c}}$ (force per apparent contact area) vs. preload for flat punch-shaped pillars with a radius of $r=25 \mu \mathrm{m}$ and an aspect ratio of 1 .

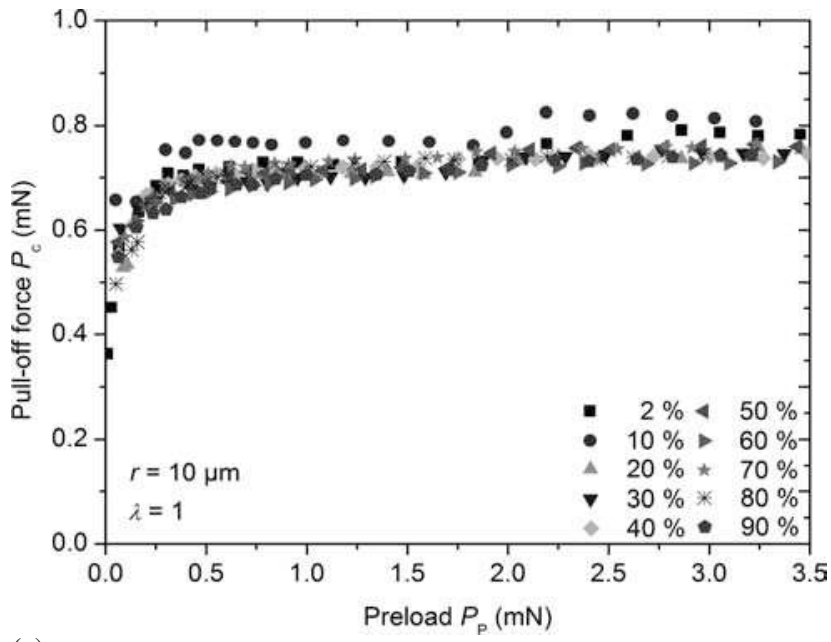

(a)

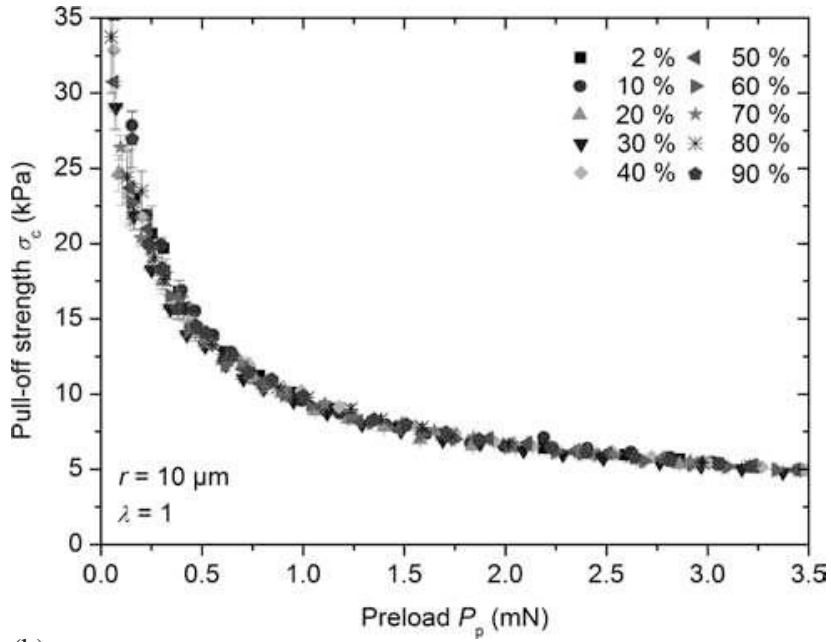

(b)
You will find the article and additional material by entering the document number MK110146 on our website at www.ijmr.de

Fig. A2. Same as Fig. A1 for flat punch-shaped pillars with a radius of $r=10 \mu \mathrm{m}$ and an aspect ratio of 1 . 


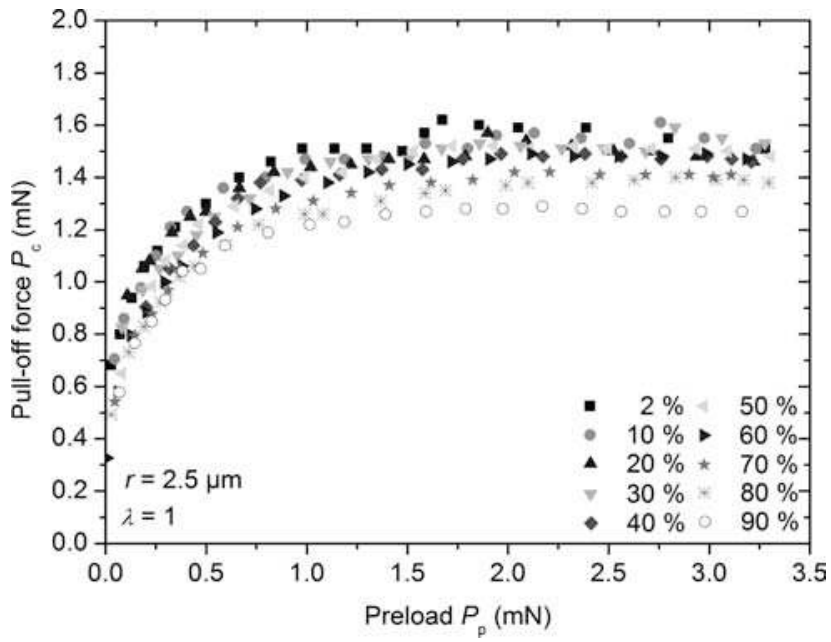

(a)

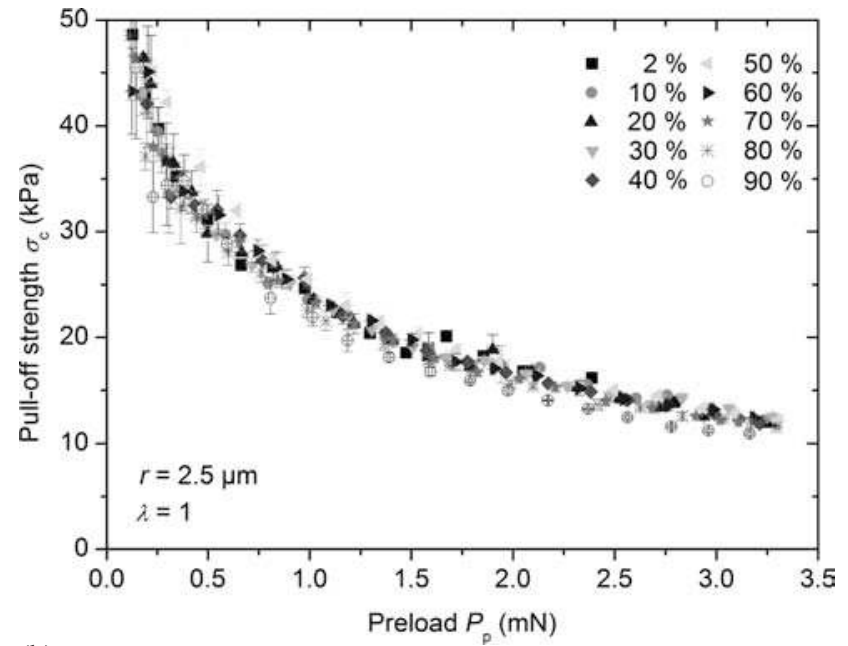

(b)

Fig. A3. Same as Fig. A1 for flat punch-shaped pillars with a radius of $r=2.5 \mu \mathrm{m}$ and an aspect ratio of 1 .

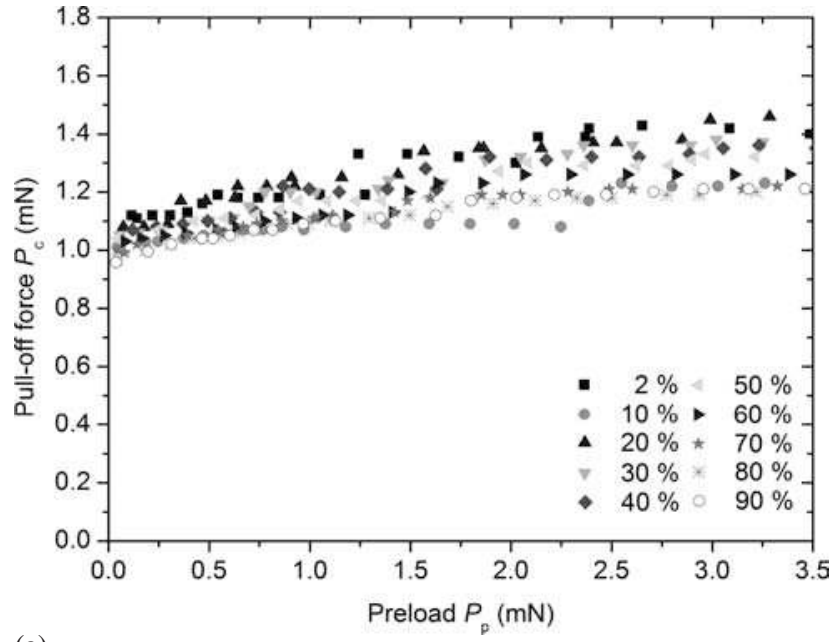

(a)

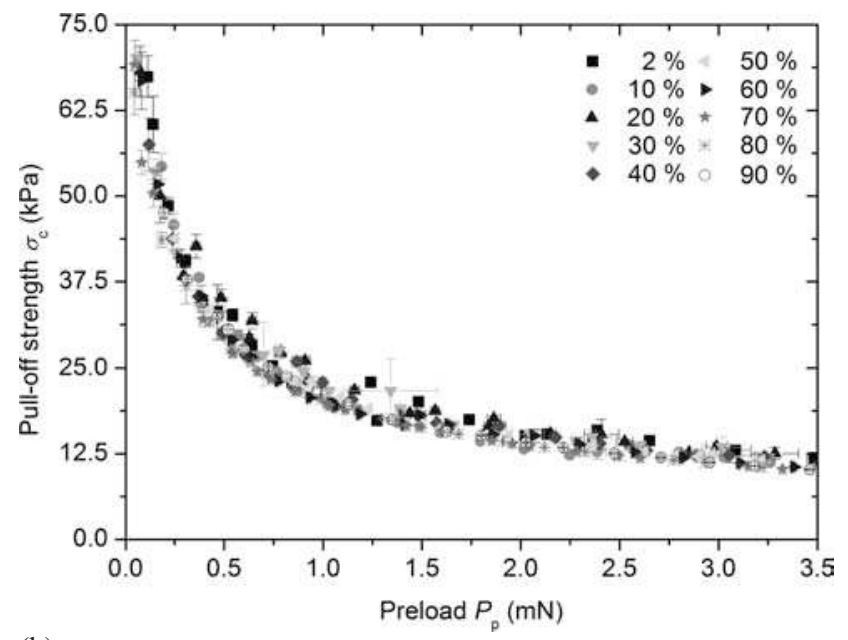

(b)

Fig. A4. Effect of ambient humidity on adhesion: (a) Pull-off force vs. preload, (b) pull-off strength $\sigma_{\mathrm{c}}$ (force per apparent contact area) vs. preload for flat, unstructured PDMS.

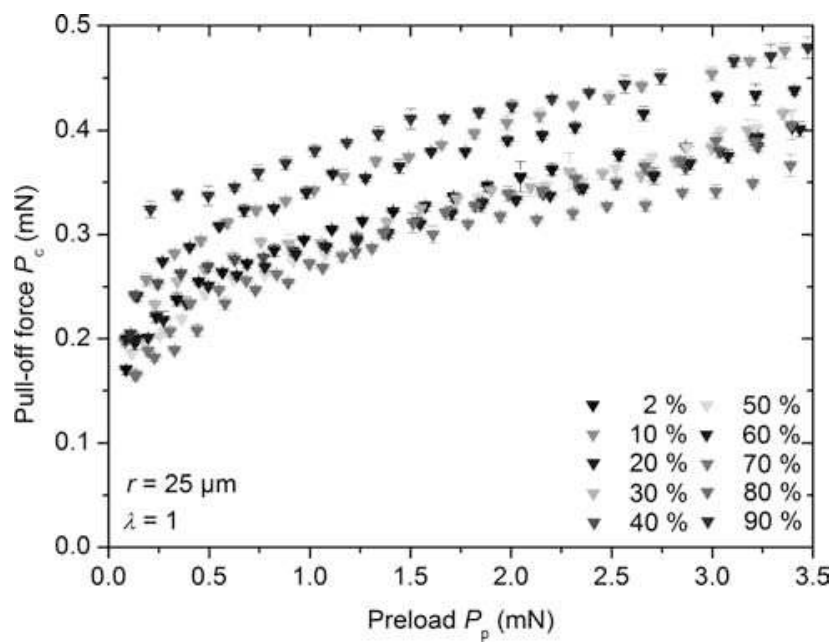

(a)

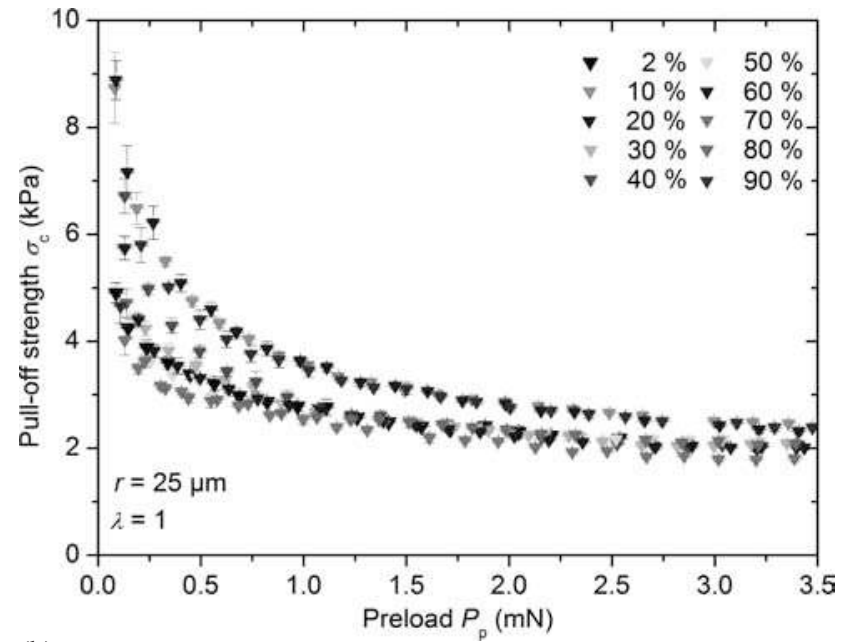

(b)

Fig. A5. Same as Fig. A4 for pillars with hemispherical tip shape having a radius of $r=25 \mu \mathrm{m}$ and an aspect ratio of 1 .

Int. J. Mat. Res. (formerly Z. Metallkd.) 100 (2009) 8 
S. Buhl et al.: Humidity influence on the adhesion of biomimetic fibrillar surfaces

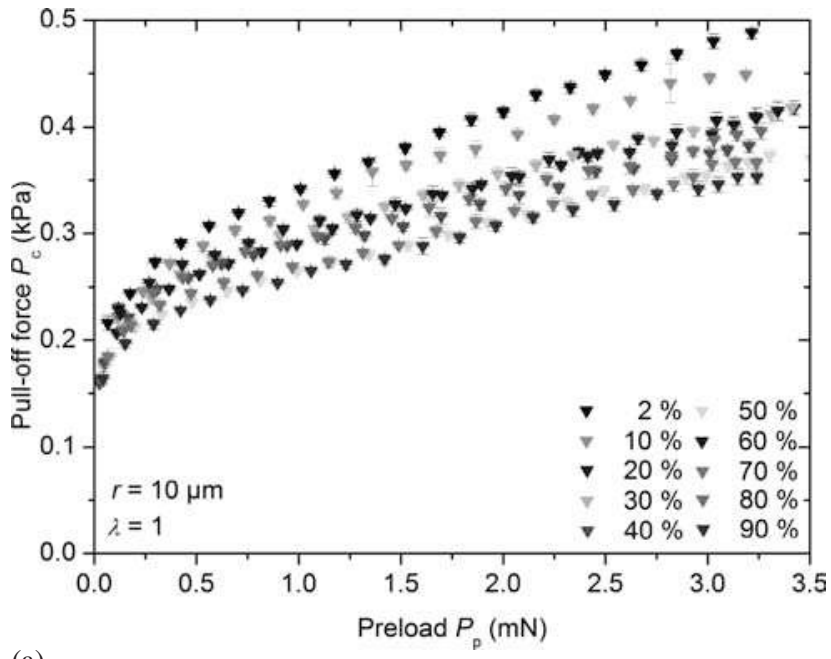

(a)

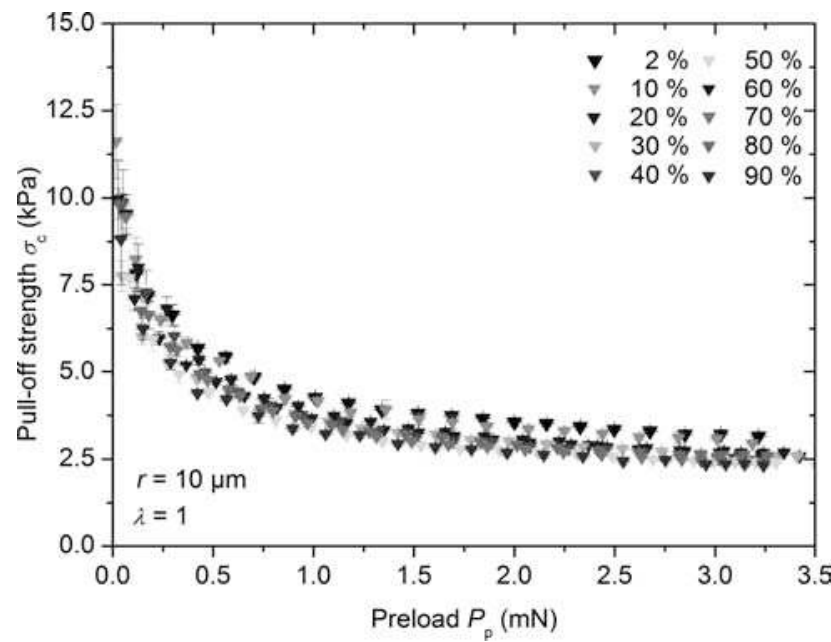

(b)

Fig. A6. Same as Fig. A4 for pillars with hemispherical tip shape having a radius of $r=10 \mu \mathrm{m}$ and an aspect ratio of 1 .

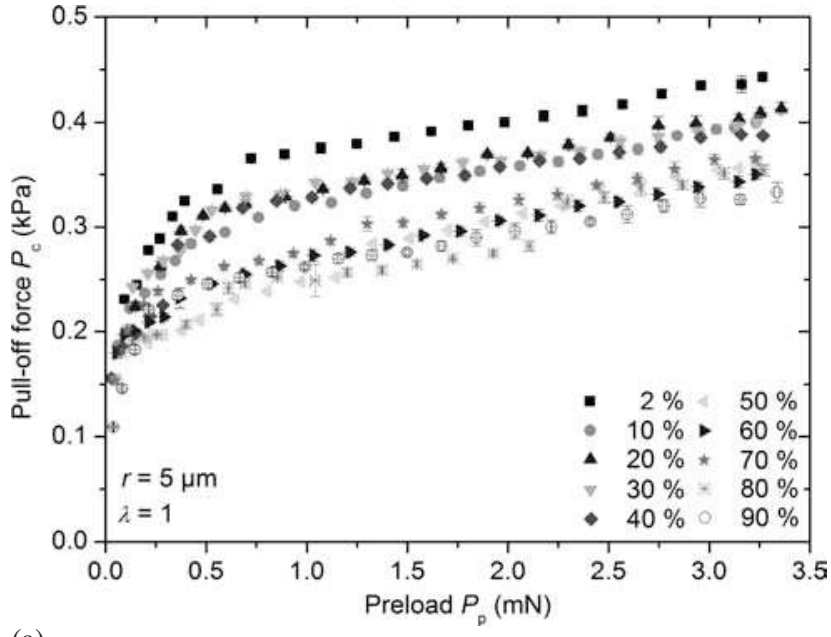

(a)

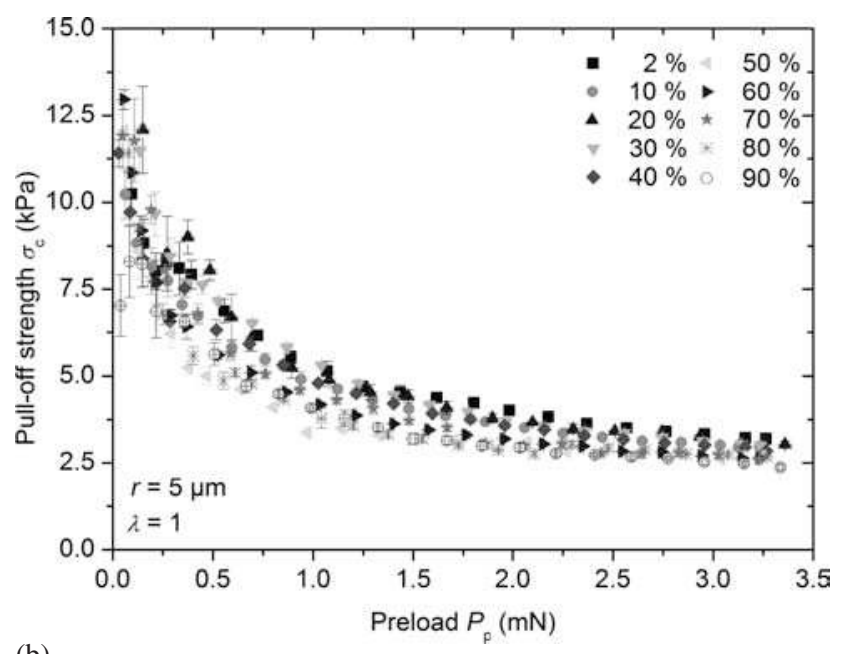

(b)

Fig. A7. Same as Fig. A4 for pillars with hemispherical tip shape having a radius of $r=5 \mu \mathrm{m}$ and an aspect ratio of 1 .

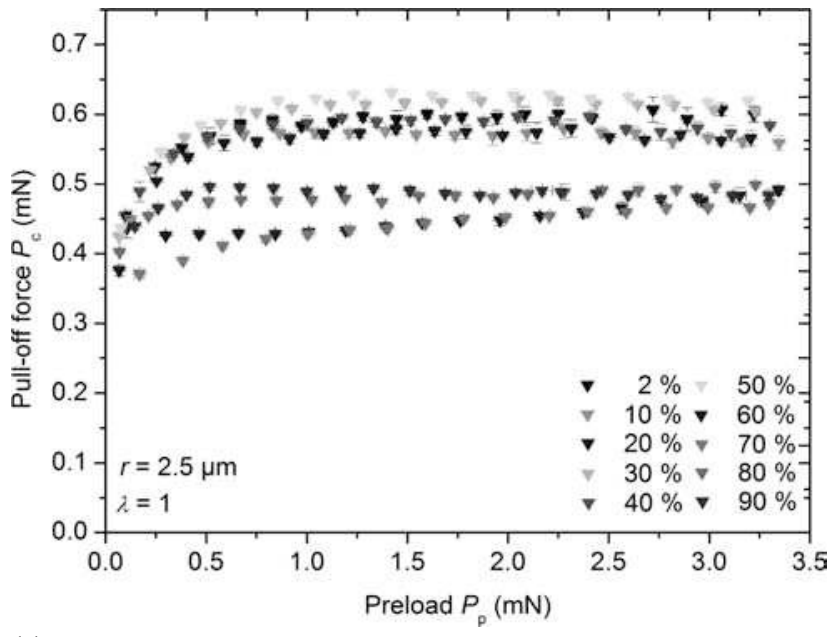

(a)

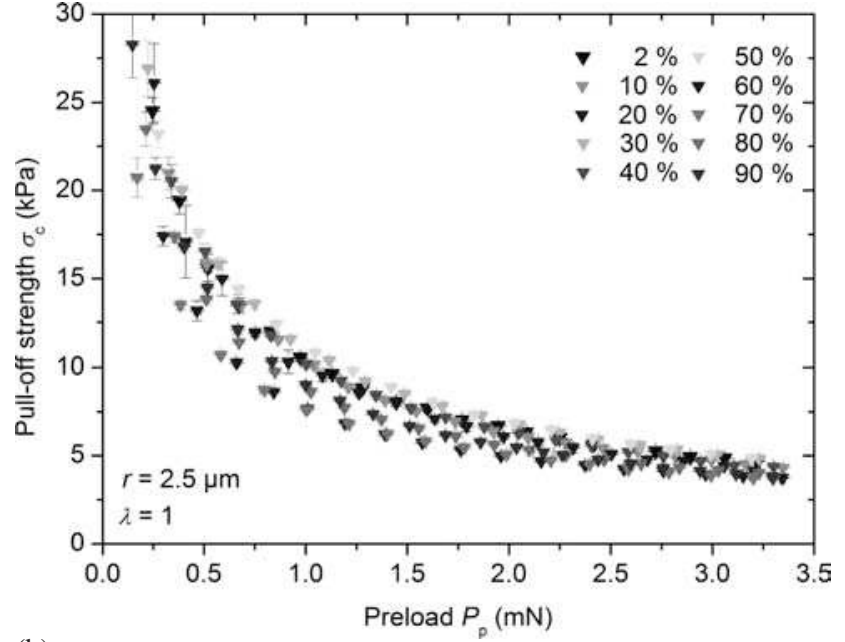

(b)

Fig. A8. Same as Fig. A4 for pillars with hemispherical tip shape having a radius of $r=2.5 \mu \mathrm{m}$ and an aspect ratio of 1 . 